\title{
Anti-Diarrheal Effects of Wood Creosote, Seirogan, in Japanese Patients
}

\author{
Masanori Takagi ${ }^{1}$, Masafumi Ito ${ }^{2, *}$, Hirofumi Morino ${ }^{3}$, Takanori Miura ${ }^{3}$, Kyoichi Oshida $^{3}$, \\ Mayu Suzuki ${ }^{4}$, Hiroshi Takemori ${ }^{5}$ id and Takashi Shibata ${ }^{6}$ \\ 1 United Graduate School of Drug Discovery and Medical Information Sciences, Gifu University, Yanagido 1-1, \\ Gifu 501-1193, Japan; masanori.takagi@seirogan.co.jp \\ 2 Taiko Pharmaceutical Co., Ltd., Uchihonmachi 3-34-14, Suita, Osaka 564-0032, Japan \\ 3 R\&D Center, Taiko Pharmaceutical Co., Ltd., 1-2-1 Hikaridai, Seikacho, Sorakugun, Kyoto 619-0237, Japan; \\ morino@seirogan.co.jp (H.M.); miura@seirogan.co.jp (T.M.); kyoichi.oshida@seirogan.co.jp (K.O.) \\ 4 Department of Chemistry and Biomolecular Science, Faculty of Engineering, Gifu University, Yanagido 1-1, \\ Gifu 501-1193, Japan; w3032081@edu.gifu-u.ac.jp \\ 5 United Graduate School of Drug Discovery and Medical Information Sciences, Gifu University, Yanagido 1-1, \\ Gifu 501-1193, Japan; htake@gifu-u.ac.jp \\ 6 Taiko Pharmaceutical Co., Ltd., Nishihonmachi 1-4-1, Nishiku, Osaka 564-0032, Japan; \\ t.shibata@seirogan.co.jp \\ * Correspondence: masafumi.itoh@seirogan.co.jp; Tel.: +81-6-6382-1076
}

Received: 7 September 2019; Accepted: 9 December 2019; Published: 10 December 2019

check for updates

\begin{abstract}
Wood creosote, Seirogan, is a non-prescription drug used to treat diarrhea. However, reports of its clinical use are rare. Here, we report the efficacy of wood creosote (3 capsules daily) in alleviating diarrheal symptoms in 148 patients from 10 clinics in Osaka, Japan. Wood creosote was classified to be remarkably effective, effective, partially effective, or not effective on the basis of the degree of alleviation of diarrheal symptoms. The anti-diarrheal efficacy of wood creosote soft capsules did not differ between males and females. Younger patients (21-30 years) showed greater improvement in diarrhea symptoms than elderly patients ( $>61$ years) did. Wood creosote soft capsules were remarkably effective (44 patients), effective (71 patients), and partially effective (13 patients) in alleviating diarrhea symptoms due to a variety of causes. To the best of our knowledge, this is the first report on the clinical effectiveness of wood creosote soft capsules in treating diarrhea.
\end{abstract}

Keywords: diarrhea; wood creosote; Seirogan; retrospective study; soft capsule

\section{Introduction}

Wood creosote (Seirogan) in soft capsule is a Japanese traditional anti-diarrheal medication [1]. In East Asian countries, wood creosote is used as a non-prescription drug (and in the United States, as a supplement) for the treatment of patients with a variety of diarrheal symptoms [2]. Wood creosote is made from tar, which is prepared by distillation of the stems of beech trees [3]. It is a colorless or light-yellow liquid with a peculiar smell. Although wood creosote shows only a weak antibacterial effect, it shows no or very few adverse effects [4,5]. Therefore, wood creosote has been approved as a non-prescription drug. Owing to its easy-to-use nature and efficacy and safety, wood creosote has captured more than $50 \%$ share of the anti-diarrheal market in Japan. However, reports on its clinical use for the treatment of diarrhea are scarce [6]. There has been a growing emphasis on the use of drugs from natural origin, which are considered safer than synthetic ones; thus, the clinical potential of wood creosote in treating diarrhea should be explored.

Here, we summarize the cases of 148 diarrhea patients who were treated with wood creosote capsules for 1-3 days. In $>78 \%$ of cases, diarrheal symptoms were decreased by treatment with wood 
creosote capsules. To the best of our knowledge, this is the first report on the anti-diarrheal efficacy of wood creosote for a variety of diarrheal symptoms.

\section{Case Presentation}

\subsection{Subjects}

Primary care physicians in 10 clinics in Japan made the diagnosis of diarrhea. All procedures of this study were approved by the ethics committee of Taiko Pharmaceutical Co., Ltd. (No. 790716). Patients (aged 15-85 years; $n=148$ (71 males and 78 females)) were categorized into 22 groups according to their diagnoses (Table 1). The age-wise distribution of patients is shown in Table 2. Taiko Pharmaceutical Co., Ltd. covered all-expense of the treatment of patients during the survey. All doctors had no conflicts of interest in the survey. The protocol of the study was approved by the Ethics Committee of Taiko Pharmaceutical Co., Ltd. (No. 790716) and presented to individual hospitals or clinics (Miyaura Clinic, Yokota Clinic, Hotta Clinic, Terada-Manjyu Clinic, Kondo Clinic, Yasui Clinic, Ajinomoto Clinic, Toyama Red Cross Hospital, Osaka Gyomei-kan Hospital, and Asahi Osaka Clinic, see details in Supplementary materials. An original protocol written in Japanese and all data were submitted to "data".). According to the original protocol and contracts, we re-confirmed that all subjects had given their informed consent for inclusion prior to their participation in the study. The study had been conducted in accordance with the Declaration of Helsinki, which was re-approved by the Ethics Committee of Taiko Pharmaceutical Co., Ltd. (No. 190927).

Table 1. Number of patients and causes of diarrhea at diagnosis.

\begin{tabular}{cccc}
\hline Diagnosis. & No. of Patients & Diagnosis & No. of Patients \\
\hline Acute enteritis & 71 & Colitis & 3 \\
Infectious gastroenteritis & 14 & Chronic gastroenteritis & 2 \\
Uncomplicated diarrhea & 12 & Suspicion of food poisoning & 2 \\
Acute gastroenteritis & 7 & Chronic colitis & 1 \\
Acute colitis & 6 & Chronic diarrhea & 1 \\
Chronic enteritis & 6 & Nervous gastroenteritis & 1 \\
Irritable colitis & 5 & Habitual diarrhea & 1 \\
Gastrogenic diarrhea & 3 & Catarrhal colitis & 1 \\
Nervous diarrhea & 3 & Hemorrhagic gastritis & 1 \\
Gastroenteritis & 3 & Gastric ulcer & 1 \\
Ulcerous colitis & 3 & Acute pancreatitis & 1 \\
& & Total & 148 \\
\hline
\end{tabular}

Table 2. Age-wise distribution of patients.

\begin{tabular}{cc}
\hline Age (Years) & Number \\
\hline$\leq 20$ & 6 \\
$21-30$ & 30 \\
$31-40$ & 23 \\
$41-50$ & 22 \\
$51-60$ & 31 \\
$\geq 61$ & 36 \\
Total & 148 \\
\hline
\end{tabular}

\subsection{Treatments}

Because wood creosote has a typical smell, oral treatment with raw wood creosote is intolerable for most patients. Therefore, $166 \mathrm{mg}$ of wood creosote was adsorbed onto $66 \mathrm{mg}$ of magnesium aluminometasilicate and packed into capsules. The patients took one capsule after a meal (total three capsules per day) for 1-3 days. When patients were unable to take meals, they took wood creosote capsules at 9:00, 12:00, and 18:00. The patients who received other related medications, such as 
berberine-containing medicines, antibiotics, synthetic antibacterial agents, or antiflatulents, during the treatment with wood creosote capsules were excluded from this study. Pregnant patients were not recruited in this study.

\subsection{Evaluation}

Records of fecal score, frequency of defecation per day, and other symptoms before and after the treatment with wood creosote were documented using a score sheet (see Supplementary materials, Score sheet). Finally, primary care physicians evaluated the efficacy of wood creosote capsules as follows:

(1) Remarkably effective: diarrheal symptoms disappeared within 3 days after treatment initiation. Stool consistency was normalized, and the patients felt comfortable.

(2) Effective: diarrheal symptoms improved within 3 days after treatment initiation. Stool consistency improved, and patients had no problem in performing daily activities.

(3) Partially effective: diarrheal symptoms partially improved within 3 days after treatment initiation. Stool consistency slightly improved.

(4) Not effective: symptoms persisted even 4 days after treatment initiation.

No data could be subjected to statistical analyses.

\subsection{Results}

A summary of the efficacy of wood creosote capsules on diarrheal symptoms is shown in Table 3. In more than $78 \%$ of the patients, diarrheal symptoms were improved effectively (remarkably effective + effective). No difference was observed in the anti-diarrheal efficacy of wood creosote capsules between sexes (Figure 1).

Table 3. Summary of the efficacy of wood creosote capsules in the treatment of diarrhea.

\begin{tabular}{ccc}
\hline & Number & $\%$ \\
\hline Remarkably effective & 44 & 29.73 \\
Effective & 72 & 48.65 \\
Partially effective & 13 & 8.78 \\
Not effective & 19 & 12.84 \\
Total & 148 & 100 \\
\hline
\end{tabular}

Male

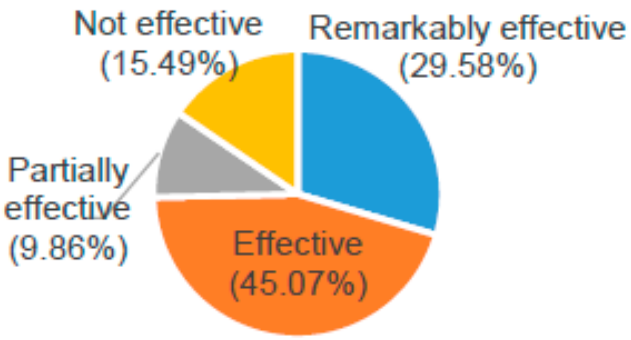

Female

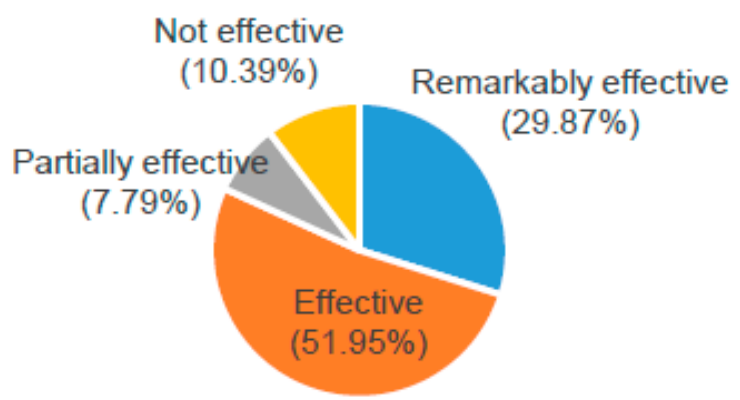

Figure 1. Comparison of the anti-diarrheal efficacy of wood creosote capsules between male $(n=71)$ and female $(n=77)$ patients.

Next, we examined the anti-diarrheal efficacy of wood creosote capsules according to the age of the patients (Figure 2). Younger patients (21-30 years) showed a greater improvement of the diarrhea symptoms compared with elderly patients ( $>61$ years). 


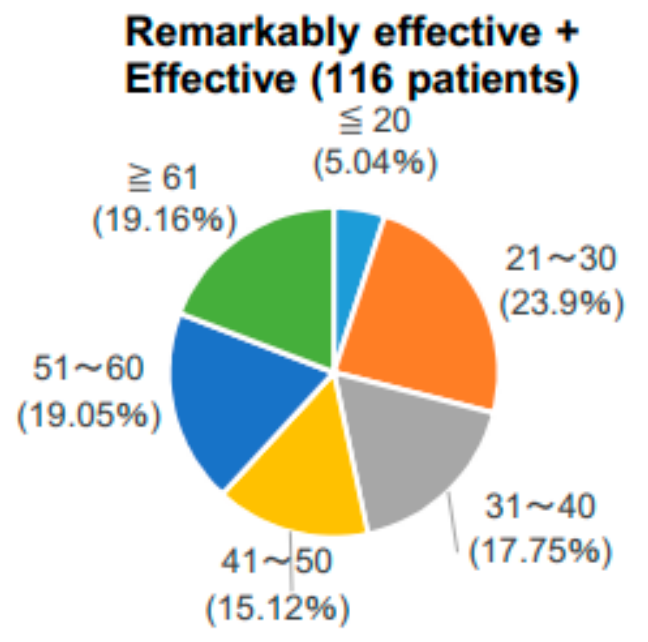

\section{Partially effective + Not effective (32 patients)}

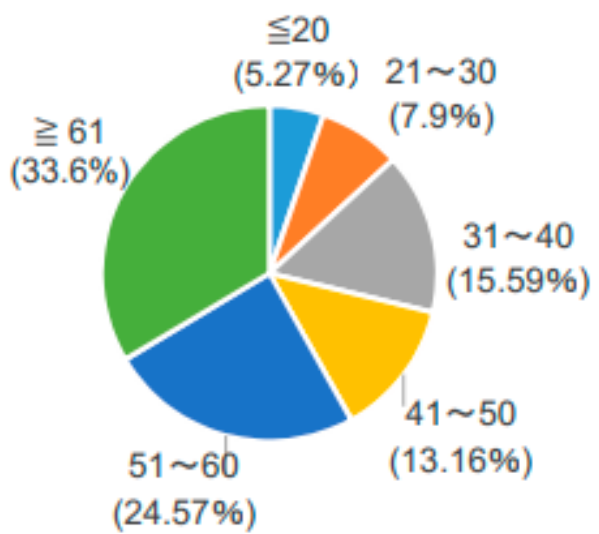

Figure 2. Anti-diarrheal efficacy of wood creosote soft capsules in younger patients. Patients were classified according to their age and improvement rate (\%), which was calculated for the total number of patients (116 for remarkably effective + effective or 32 for partially effective + not effective) in each group.

The number of patients and the corresponding improvement rate (\%) for each diarrheal cause are summarized in Table 4. Wood creosote capsules were effective in patients with acute enteritis, uncomplicated diarrhea, infectious gastroenteritis, and irritable colitis. In addition, wood creosote capsules were also effective in patients with nervous diarrhea and irritable colitis. The efficacy of wood creosote was re-evaluated after a classification based on diarrheal causes and is shown in Figure 3.

Table 4. Efficacy of wood creosote capsules against each diarrheal cause.

\begin{tabular}{|c|c|c|c|c|c|c|c|c|}
\hline \multirow[b]{2}{*}{ Diagnosis } & \multicolumn{8}{|c|}{ Number of Patients } \\
\hline & $\begin{array}{c}\text { Remarkably } \\
\text { Effective }\end{array}$ & $(\%)$ & Effective & $(\%)$ & $\begin{array}{l}\text { Partially } \\
\text { Effective }\end{array}$ & $(\%)$ & $\begin{array}{c}\text { Not } \\
\text { Effective }\end{array}$ & $(\%)$ \\
\hline Acute enteritis & 25 & 35.21 & 30 & 42.25 & 8 & 11.27 & 8 & 11.27 \\
\hline Acute gastroenteritis & 1 & 14.29 & 5 & 71.43 & 0 & 0 & 1 & 41.29 \\
\hline Acute colitis & 1 & 16.67 & 5 & 83.33 & 0 & 0 & 0 & 0 \\
\hline Chronic enteritis & 0 & 0 & 3 & 50 & 2 & 33.33 & 1 & 16.67 \\
\hline Chronic gastroenteritis & 0 & 0 & 0 & 0 & 1 & 50 & 1 & 50 \\
\hline Chronic colitis & 0 & 0 & 0 & 0 & 0 & 0 & 1 & 100 \\
\hline Chronic diarrhea & 0 & 0 & 1 & 100 & 0 & 0 & 0 & 0 \\
\hline Infectious gastroenteritis & 2 & 14.29 & 10 & 71.43 & 1 & 7.14 & 1 & 7.14 \\
\hline Uncomplicated diarrhea & 9 & 75 & 3 & 25 & 0 & 0 & 0 & 0 \\
\hline Irritable colitis & 3 & 60 & 2 & 40 & 0 & 0 & 0 & 0 \\
\hline Gastrogenic diarrhea & 0 & 0 & 3 & 100 & 0 & 0 & 0 & 0 \\
\hline Nervous diarrhea & 2 & 66.67 & 1 & 33.33 & 0 & 0 & 0 & 0 \\
\hline Gastroenteritis & 1 & 33.33 & 1 & 33.33 & 0 & 0 & 1 & 33.33 \\
\hline Nervous gastroenteritis & 0 & 0 & 1 & 100 & 0 & 0 & 0 & 0 \\
\hline Ulcerous colitis & 0 & 0 & 2 & 66.57 & 0 & 0 & 1 & 33.3 \\
\hline Habitual diarrhea & 0 & 0 & 1 & 100 & 0 & 0 & 0 & 0 \\
\hline Catarrhal colitis & 0 & 0 & 1 & 100 & 0 & 0 & 0 & 0 \\
\hline Colitis & 0 & 0 & 1 & 33.33 & 0 & 0 & 2 & 66.67 \\
\hline Hemorrhagic gastritis & 0 & 0 & 0 & 0 & 0 & 0 & 1 & 100 \\
\hline Gastric ulcer & 0 & 0 & 0 & 0 & 1 & 100 & 0 & 0 \\
\hline Acute pancreatitis & 0 & 0 & 1 & 100 & 0 & 0 & 0 & 0 \\
\hline Suspicion of food poisoning & 0 & 0 & 1 & 50 & 0 & 0 & 1 & 50 \\
\hline Total number & 44 & & 72 & & 13 & & 19 & \\
\hline
\end{tabular}




\section{Acute enteritis (71)}

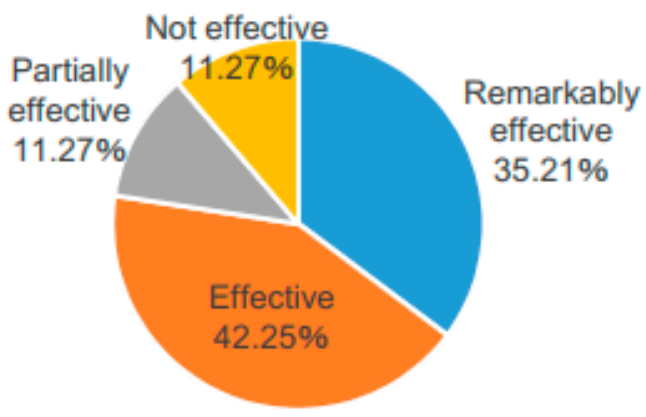

\section{Infectious gastroenteritis (14) + gastroenteritis (3)}

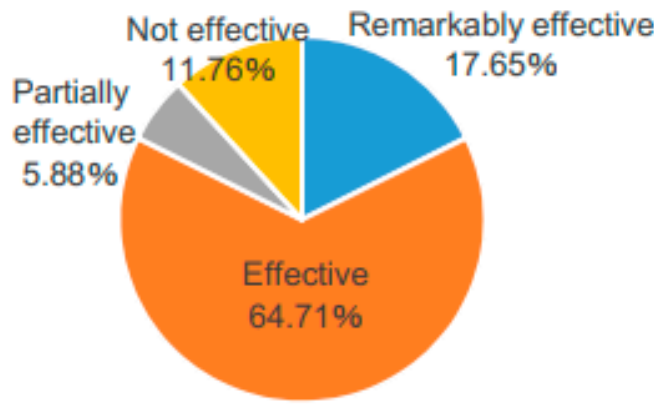

Ulcerous colitis (3)

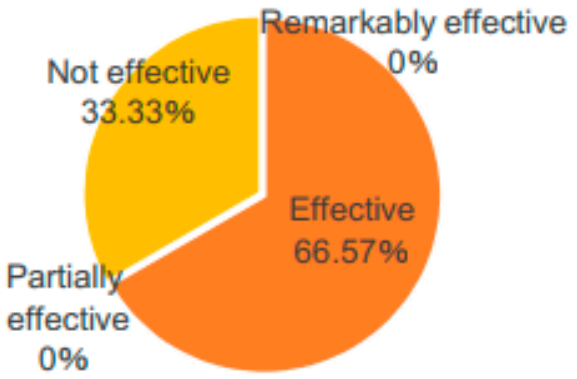

\section{Uncomplicated diarrhea (12) + habitual diarrhea (1)}

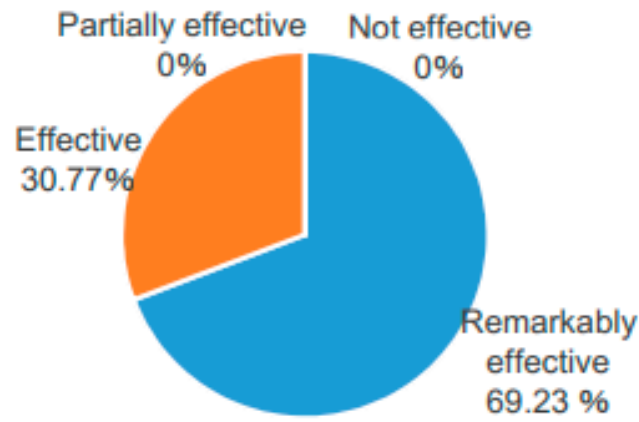

\section{Irritable colitis (5) + nervous diarrhea (3)}

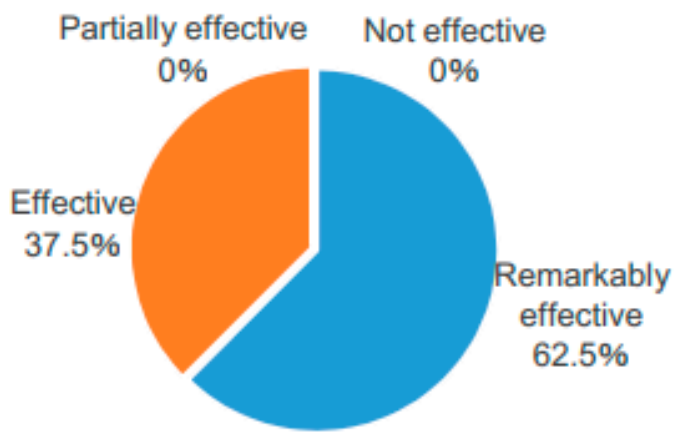

\section{Others (36)}

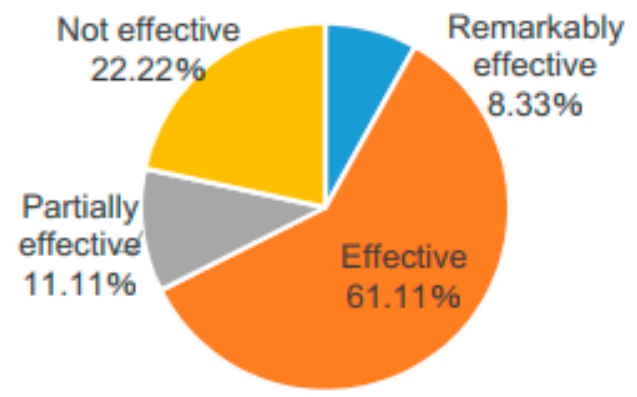

Figure 3. Re-evaluation of the efficacy of wood creosote capsules after classifying the patients on the basis of diarrheal causes. Numbers in brackets indicate the number of patients.

Wood creosote capsules efficiently decreased diarrheal symptoms (frequency of defecation and fecal score) due to different causes. Although some patients did not respond to the treatment, no specific cause of ineffectiveness was found. Some patients experienced adverse effects: bloating sensation (one patient), nausea (one patient), and cardialgia (one patient). Except for one patient, however, these adverse effects disappeared during the treatment, without abrupt withdrawal of the medication.

\section{Discussion}

Wood creosote (capsules) was remarkably effective (44 patients), effective (71 patients), and partially effective (13 patients) in alleviating diarrhea symptoms. Although younger patients (21-30 years) showed greater improvement than elderly patients ( $>61$ years), wood creosote capsules showed 
anti-diarrheal effects in all patients. Notably, they were more effective against acute diarrhea than chronic diarrhea. In addition, the present survey suggests that wood creosote could be beneficial for patients with a wide range of diarrheal symptoms. Especially, this medication shows high efficacies against mild to moderate symptoms, but not for the treatment of organic disorders.

Wood creosote, referred to as medicinal creosote, is called Seirogan (a trade name) in Japan [1]. Wood creosote is a mixture of phenolic compounds (including creosol, o-cresol, guaiacol, and 4-ethylguaiacol), which are the major components responsible for its anti-diarrheal activities [2]. Wood creosote normalizes bowel peristalsis and suppresses excess water leakage from epithelial cells [3]. Moreover, it inhibits L-type $\mathrm{Ca}^{2+}$ channels, which lowers the $\mathrm{Ca}^{2+}$ influx in smooth muscle cells. The decrease in $\mathrm{Ca}^{2+}$ ion levels suppresses smooth muscle contraction, leading to the normalization of bowel peristalsis [4].

Moreover, wood creosote has been reported to directly inhibit the $\mathrm{Cl}^{-}$channel, cystic fibrosis transmembrane conductance regulator (CFTR) on the apical membrane. The inhibition of the $\mathrm{Cl}^{-}$ channel induces an osmolality change, which suppresses water leakage [5]. Because $\mathrm{Cl}^{-}$channel hyper-activity is associated with different types of diarrhea [6], wood creosote may be effective against a wide range of diarrheal symptoms. The use of wood creosote was less effective against diarrheal symptoms caused by organic disorders in the digestive tract, such as colitis, as compared with those associated with $\mathrm{Cl}^{-}$channel-related diarrhea, suggesting that $\mathrm{Cl}^{-}$channels may be the target of wood creosote.

Besides modulating these ion channel-mediated effects, wood creosote has been reported to prevent hyper colonic motility caused by the 5-HT3 receptor and the 5-HT4 receptor in the proximal colon and distal colon, respectively [7]. Wood creosote has been shown to prevent the increase in colonic motility induced by stress stimulation via 5 -HT receptors in the colon, suggesting that it might be effective in treating stress-induced diarrhea with abdominal pain [8].

Loperamide, an opioid receptor agonist, is used to treat acute diarrhea worldwide. It also shows high efficacy against a variety of diarrheal symptoms, except those of infectious diarrhea. A double-blind study showed that the anti-diarrheal efficacy of wood creosote was almost similar to that of loperamide [9]. Besides improving other symptoms of diarrhea, wood creosote improved or resolved abdominal cramping, whereas loperamide only improved diarrhea. This suggests that wood creosote could treat diarrhea with a wider range of symptoms than loperamide [10], although further detailed comparative tests are needed.

\section{Conclusions}

Wood creosote capsules are effective in treating diarrhea due to various causes. To the best of our knowledge, this is the first report on the efficacy of wood creosote capsules against diarrhea in clinical practice.

Supplementary Materials: The following are available online at http://www.mdpi.com/2571-841X/2/4/28/s1.

Author Contributions: M.T. and M.I. wrote this manuscript, H.M., T.M., and K.O. corrected data, M.S. and H.T. obtained the data. T.S. designed this study.

Funding: No funder in this study. All research expenses were borne by Taiko Pharmaceutical Co., Ltd.

Conflicts of Interest: M.T., M.I., H.M., T.M., K.O., T.S. are employed by Taiko Pharmaceutical Co., Ltd. M.S. and H.T. have nothing to declare.

\section{References}

1. Kuge, T.; Shibata, T.; Willett, M.S. Wood creosote, the principal active ingredient of seirogan, an herbal antidiarrheal medicine: A single-dose, dose-escalation safety and pharmacokinetic study. Pharmacotherapy 2003, 23, 1391-1400. [CrossRef]

2. Ogata, N.; Baba, T. Analysis of beechwood creosote by gas chromatography-mass spectrometry and high-performance liquid chromatography. Res. Commun. Chem. Pathol. Pharmacol. 1989, 66, 411-423. 
3. Morino, H.; Ataka, K.; Ito, M.; Kuge, T. Wood creosote inhibits calcium mobilization in Guinea pig colonic smooth muscle. Biol. Pharm. Bull. 2004, 27, 1046-1051. [CrossRef] [PubMed]

4. Shi, X.Z.; Sarna, S.K. Gene therapy of Cav1.2 channel with VIP and VIP receptor agonists and antagonists: A novel approach to designing promotility and antimotility agents. Am. J. Physiol. Gastrointest. Liver Physiol. 2008, 295, G187-G196. [CrossRef] [PubMed]

5. Ogata, N.; Shibata, T. Inhibition of rat intestinal $\mathrm{Cl}^{-}$secretion by 4,5-dimethylresorcinol. Pharmacology 2004, 72, 247-253. [CrossRef] [PubMed]

6. Yibcharoenporn, C.; Chusuth, P.; Jakakul, C.; Rungrotmongkol, T.; Chavasiri, W.; Muanprasat, C. Discovery of a novel chalcone derivative inhibiting CFTR chloride channel via AMPK activation and its anti-diarrheal application. J. Pharmacol. Sci. 2019. [CrossRef] [PubMed]

7. Ataka, K.; Kuge, T.; Fujino, K.; Takahashi, T.; Fujimiya, M. Wood creosote prevents CRF-induced motility via 5-HT3 receptors in proximal and 5-HT4 receptors in distal colon in rats. Auton. Neurosci. 2007, 133, 136-145. [CrossRef] [PubMed]

8. Ataka, K.; Kuge, T.; Venkova, K.; Greenwood-Van Meerveld, B. Seirogan (wood creosote) inhibits stress-induced ion secretion in rat intestinal epithelium. Dig. Dis. Sci. 2003, 48, 1303-1309. [CrossRef] [PubMed]

9. Kuge, T.; Shibata, T.; Willett, M.S. Multicenter, double-blind, randomized comparison of wood creosote, the principal active ingredient of Seirogan, an herbal antidiarrheal medication, and loperamide in adults with acute nonspecific diarrhea. Clin. Ther. 2004, 26, 1644-1651. [CrossRef] [PubMed]

10. Ogata, N.; Ataka, K.; Morino, H.; Shibata, T. Effect of wood creosote and loperamide on propulsive motility of mouse colon and small intestine. Pharmacology 1999, 59, 212-220. [CrossRef] [PubMed]

(C) 2019 by the authors. Licensee MDPI, Basel, Switzerland. This article is an open access article distributed under the terms and conditions of the Creative Commons Attribution (CC BY) license (http://creativecommons.org/licenses/by/4.0/). 